\title{
N $88-14956$
}

AIRFOIL LARGE EDDY BREAKUP DEVICES

FOR TURBULENT DRAG REDUCTION

$$
530-05
$$

117254

139

\author{
J. B. Anders
}

NASA Langley Research Center

Hampton, Virginta 


\section{Large Eddy Breakup Devices}

Large eddy breakup (LEBU) devices are thin elements (plates or airfoils) placed horizontally in a turbulent boundary layer for the purpose of modifying the turbulent structure to achieve reduced skin friction. While the exact mechanism by which LEBU devices modify the turbulence is not well understood at this time, several proposed mechanisms are currently the subject of study at a number of research institutions in this country. What is known is that these thin-element devices can produce large reductions in turbulent skin friction for extended downstream distances. The most effective LEBU geometry thus far is a tandem arrangement of plates, 6 to 10 chord lengths apart, near the edge of the turbulent boundary layer. Net drag reductions (i.e., accounting for the drag of the thin plates) of the order of 10 percent have been reported from experiments both here at Langley and the Illinois Institute of Technology under a Langley grant. The major issues considered thus far have been concerned with minimizing device drag (through the use of very thin plates) and maximizing the drag reduction performance through parametric geometry changes.
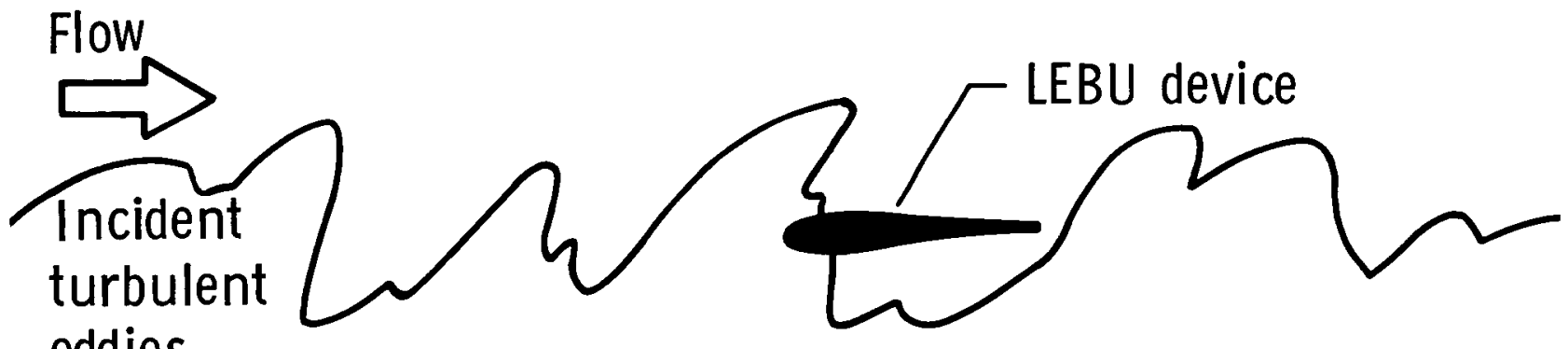

eddies

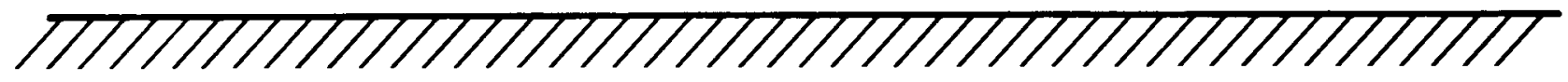

Definition of a LEBU
A device to break up and modify the large outer scales of a turbulent boundary layer for the purposes of turbulence control and drag reduction

\section{$\underline{\text { Issues }}$}

- Device drag

- Drag reduction performance 


\section{Key Application Issues for LEBU's}

While the use of thin plates to minimize device drag is a viable technique for low-speed wind tunnel experiments, it is not practical for the actual flight environment where significant structural integrity will be required. The present work was initiated to develop LEBU devices sufficiently rugged enough for flight and to determine their drag reduction performance at higher Reynolds numbers.

\section{CTOL aircraft}

- Performance at high Reynolds numbers

- Device structural integrity

- Can transonic-capable devices produce net drag reduction? 


\section{LEBU Devices}

Initial LEBU experiments were conducted using thin, flat ribbons in tandem arrays, as shown here. Second generation devices utilized flat ribbons with tapered trailing edges to reduce device drag. Currently, airfoil-shaped devices are under investigation to utilize their low-drag/higher structural stiffness characteristics.
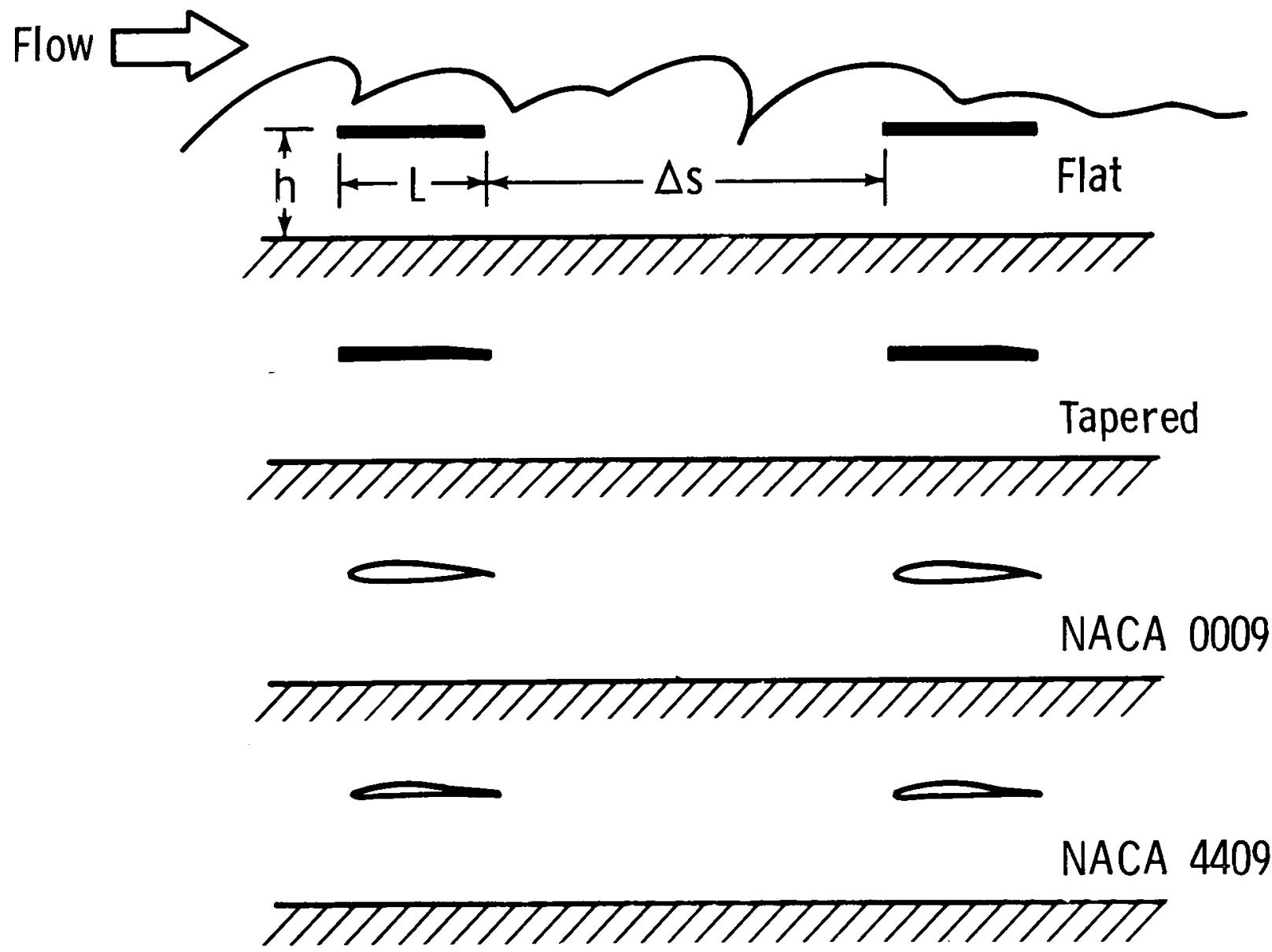


\section{Skin Friction Reduction}

The effectiveness of LEBU devices is a function of both the magnitude and the extent of the reduced $C_{f}$ region downstream. This figure shows results obtained at Langley and the Illinois Institute of Technology for thin-plate devices in a tandem arrangement. The downstream extent is shown in terms of boundary layer thicknesses $\left(\delta_{0}\right)$ from the device location $\left(x_{0}\right)$. Maximum $C_{f}$ reductions approaching 50 percent have been measured with recovery lengths greater than 100 boundary layer thicknesses. The parasite drag of the devices themselves is not included in this figure.

\section{Thin plates}

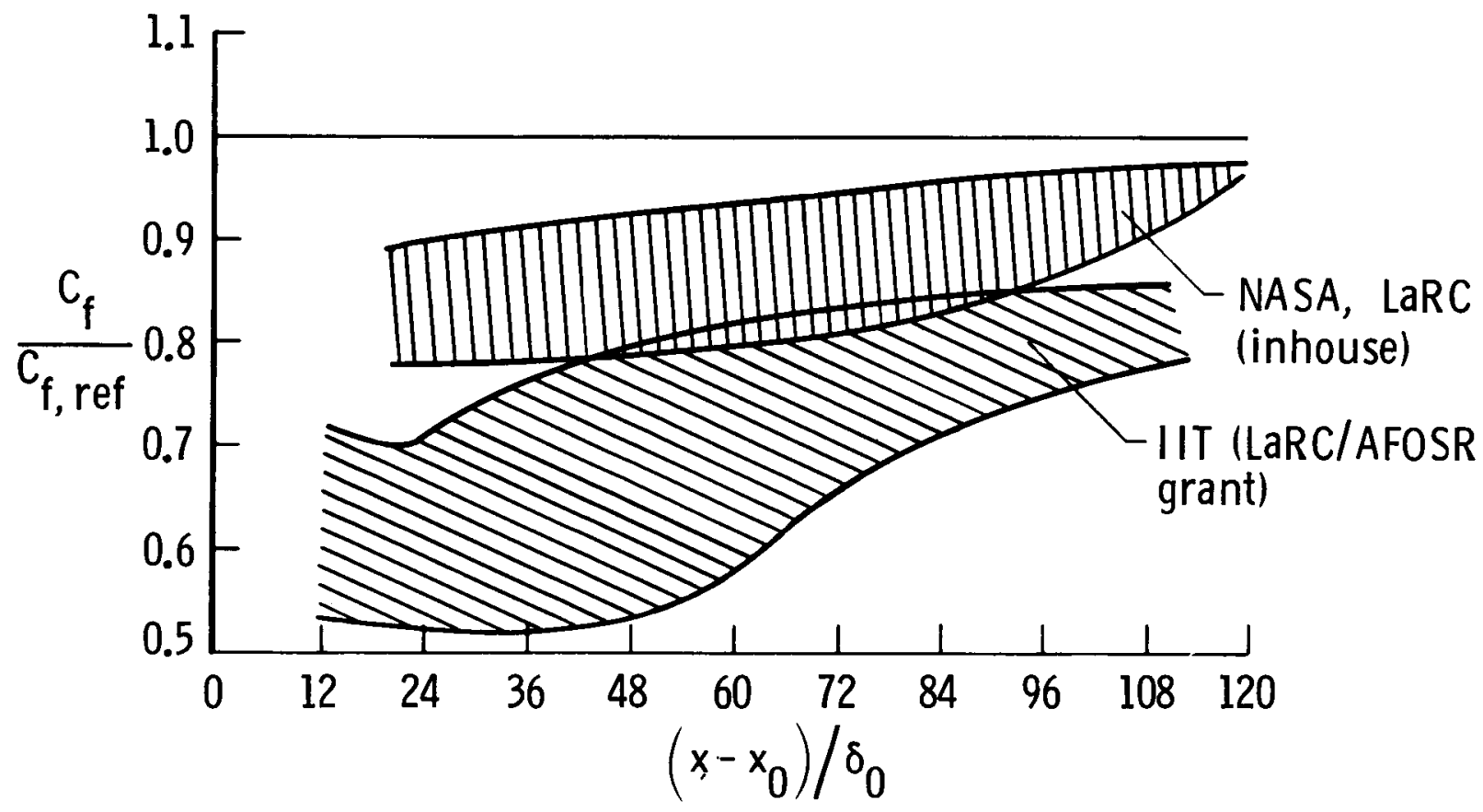


Net drag reduction is simply the downstream skin-friction drag plus the device drag of the LEBU's nondimensionalized by the reference (no device) skin-friction drag. This figure shows that the reduced skin friction downstream of LEBU devices quickly compensates for the added parasitic device drag and that net reductions $\left(0 / 0_{\text {ref }}<1\right)$ can be obtained within 30 boundary layer thicknesses downstream. Net drag reduction effectiveness is a sensitive function of device thickness, device height in the boundary layer and device chord. The spread of the data shown is a reflection of that sensitivity.

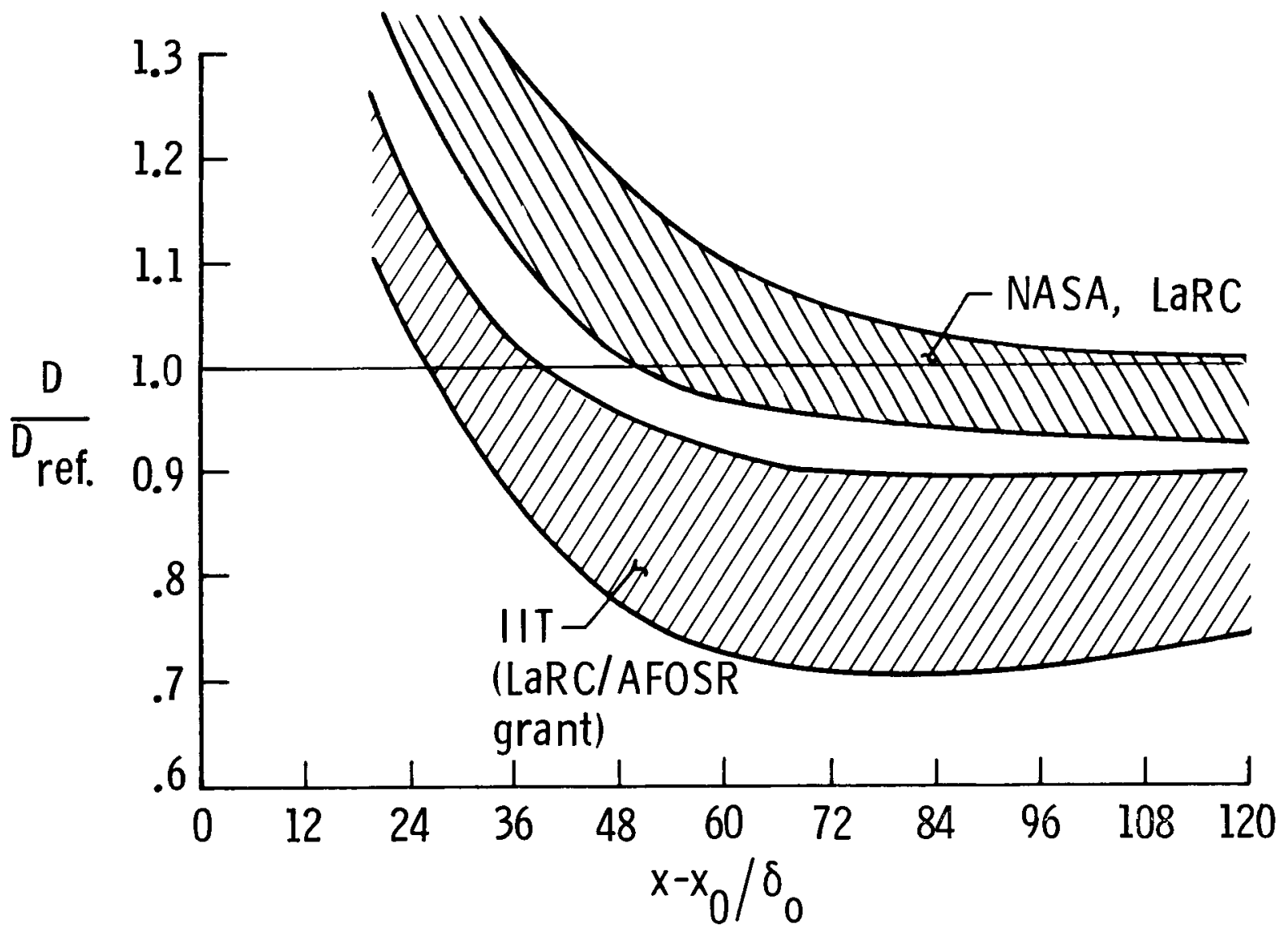




\section{Test Plate}

The present experiments were conducted on the test plate shown in this figure. The plate spanned the wind tunnel test section and the tunnel ceiling was adjusted to give a near-zero pressure gradient down the entire plate length. The LEBU devices were positioned approximately one-third of the way back from the plate leading edge where the turbulent boundary layer thickness was $25 \mathrm{~mm}$. The usable test region extended approximately $300 \mathrm{~cm}$ downstream ( 120 boundary layer thicknesses). Data was obtained in the form of velocity profiles and local skin friction was deduced from the longitudinal variation of the integral properties of the boundary layer (momentum balance).

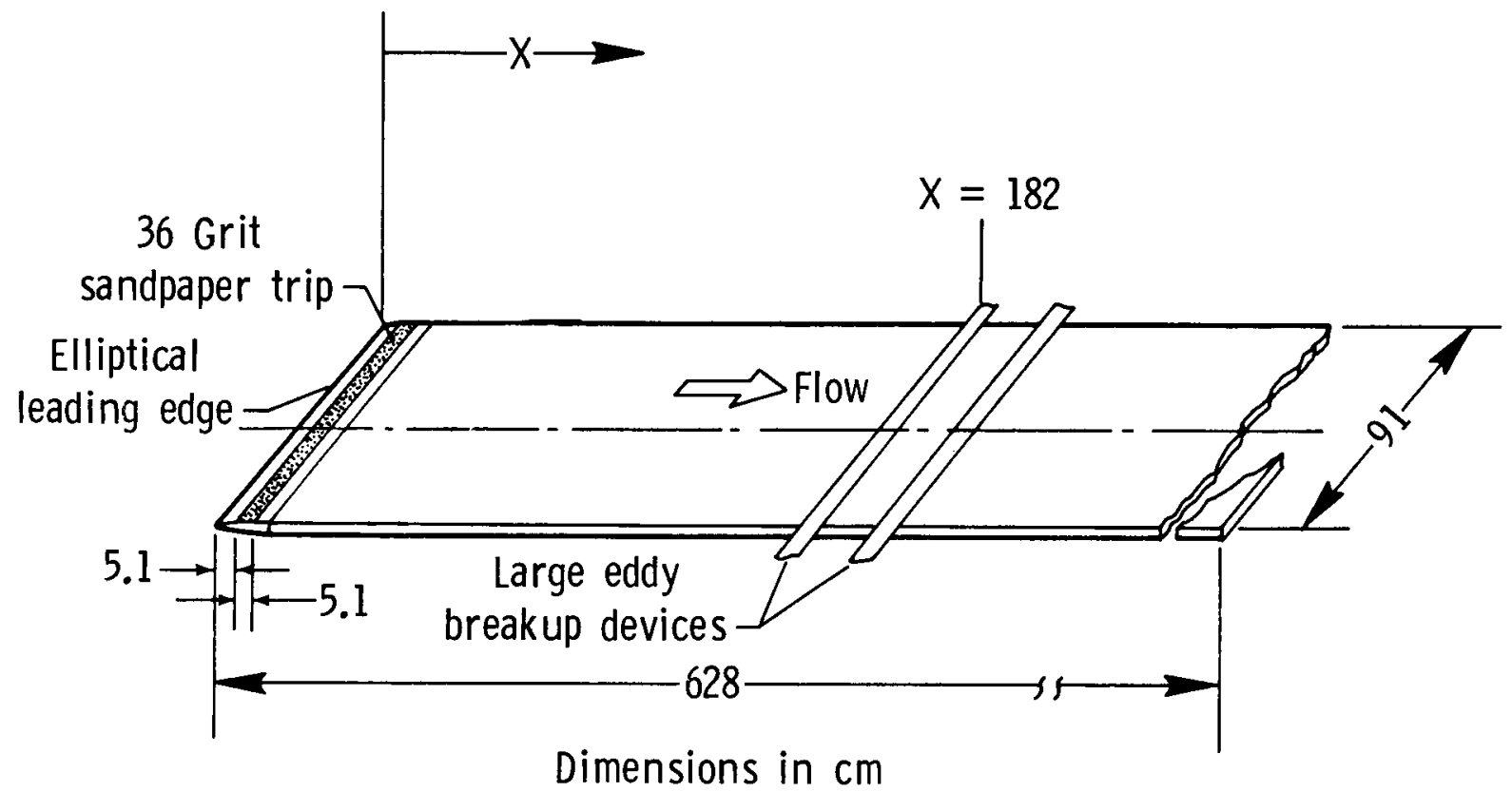


Shown in this figure are the net drag reductions obtained downstream of thin plates at both high and low Reynolds numbers. As indicated, the drag reduction performance at high Reynolds numbers is actually somewhat better, although insufficient downstream test length was available to determine the maximum net reduction obtainable for the high Reynolds number case. This gives confidence that previous, low Reynolds number results are conservative and no inherent limitations are present to prevent the application of LEBU technology to the high Reynolds number flight environment.

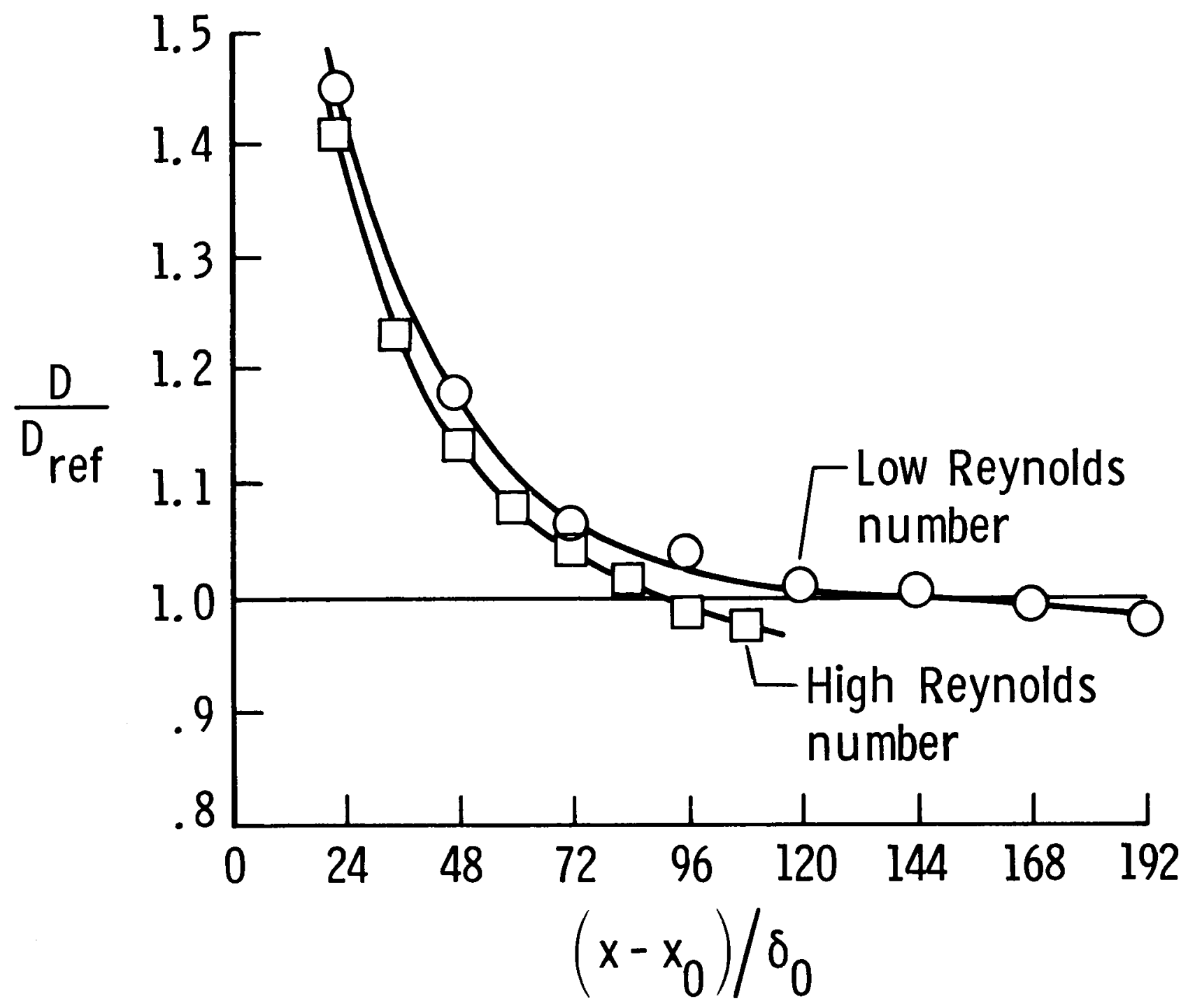




\section{Drag of Single Devices}

The device drag of thin plates (thickness/chord $\simeq .01$ ) at low Reynolds numbers is shown in this figure to be near that expected for laminar skin friction drag. Laminar skin friction drag is the theoretical minimum device drag obtainable for single elements, and hence represents a lower limit for the LEBU device drag penalty. As velocity is increased (larger $R_{l}$ ) flowinduced vibration of the thin plates becomes important and the plate thickness must be increased to provide greater stiffness. These thicker plates (thickness/chord $\simeq .02$ ) significantly increase the device drag (see thick plate data in the figure). Ultra-thin plates (thickness/chord $=.008$ ) can reduce this increased device drag but at the higher Reynolds numbers they must have closely spaced vertical supports to suppress the flow-induced vibrations. Since these vertical supports locally destroy the skin friction reduction effectiveness of the LEBU devices it is desirable to minimize the number of supports. The answer to the conflicting requirements of increased device rigidity with minimal device drag lies with airfoil-shaped devices. As shown in the figure, an NACA 0009 airfoil (thickness/chord $=.1$ ) has near laminar skin friction drag even though it is 10 times thicker and 1000 times stiffer than the thin plates.

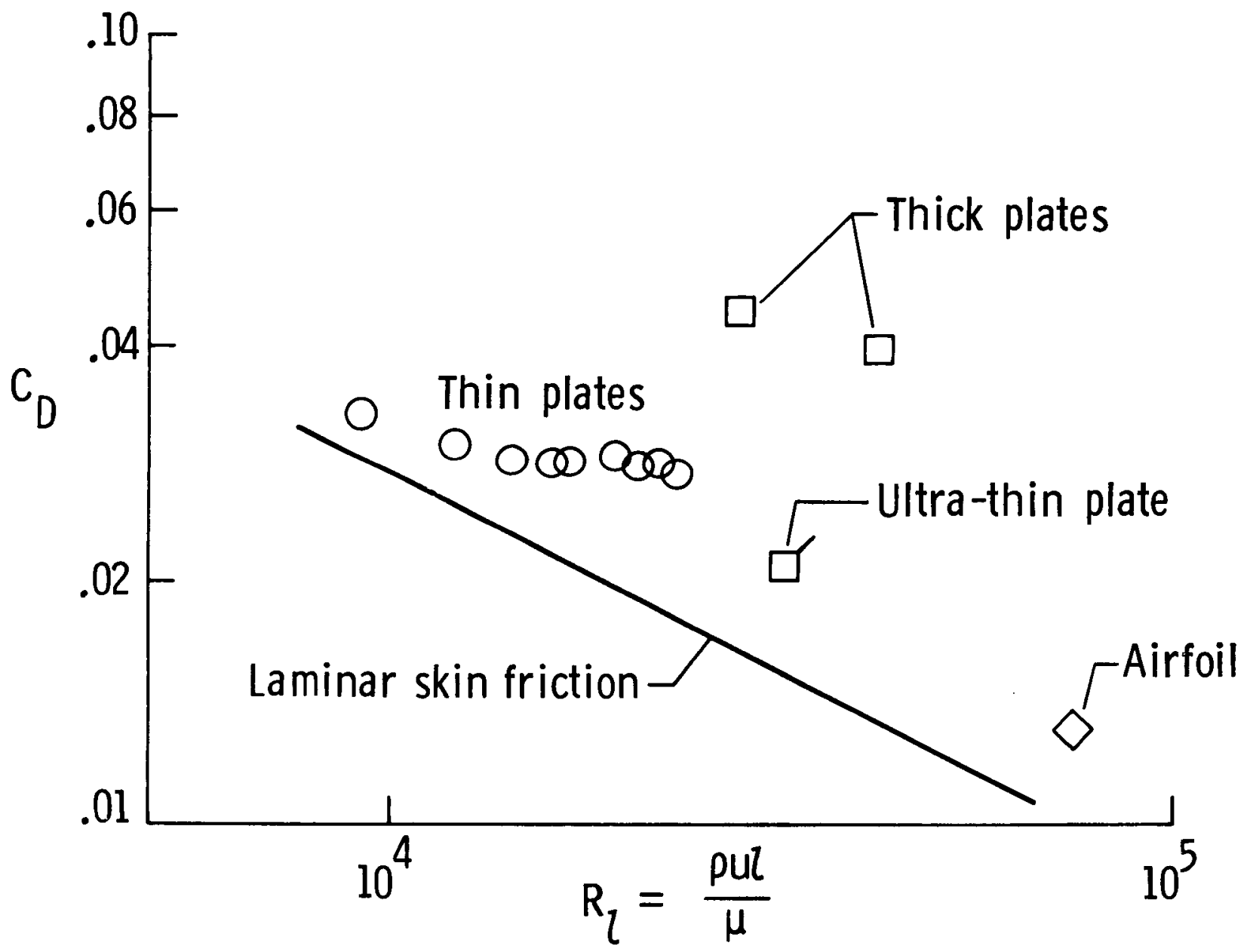




\section{Airfoil LEBU Skin Friction Reduction}

The measured, local skin friction downstream of a tandem set of NACA 0009 airfoils is shown in this figure. The maximum skin friction reduction of 30 percent occurs approximately 40 boundary layer thicknesses downstream followed by a return toward flat plate levels over the remaining 60 boundary layer thicknesses of the test region. These results compare favorably with the thin-plate results obtained previously at lower Reynolds numbers. These data indicate that airfoil-shaped devices are at least as effective in reducing skin friction as thin plates.

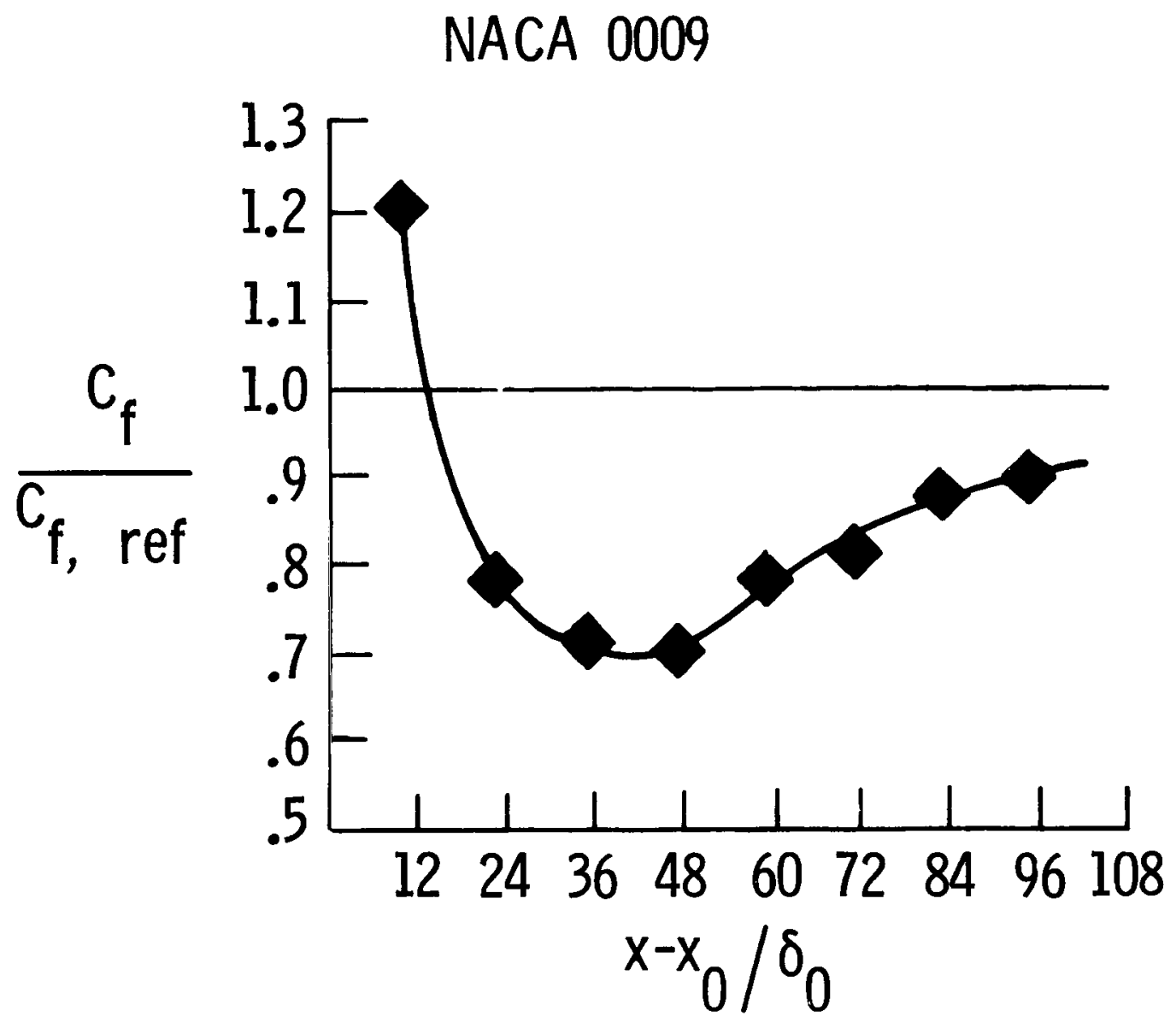


Net drag reductions obtained with tandem, NACA 0009 airfoils at high Reynolds numbers compare well with the low Reynolds number thin-plate data. The foreshortened test region for the high Reynolds number tests prevented a determination of the maximum net reduction obtainable, but it is clear from this figure that the NACA 0009 airfoils can produce net drag reductions of the order of 10 percent over approximately 100 boundary layer thicknesses.

\section{NACA 0009}

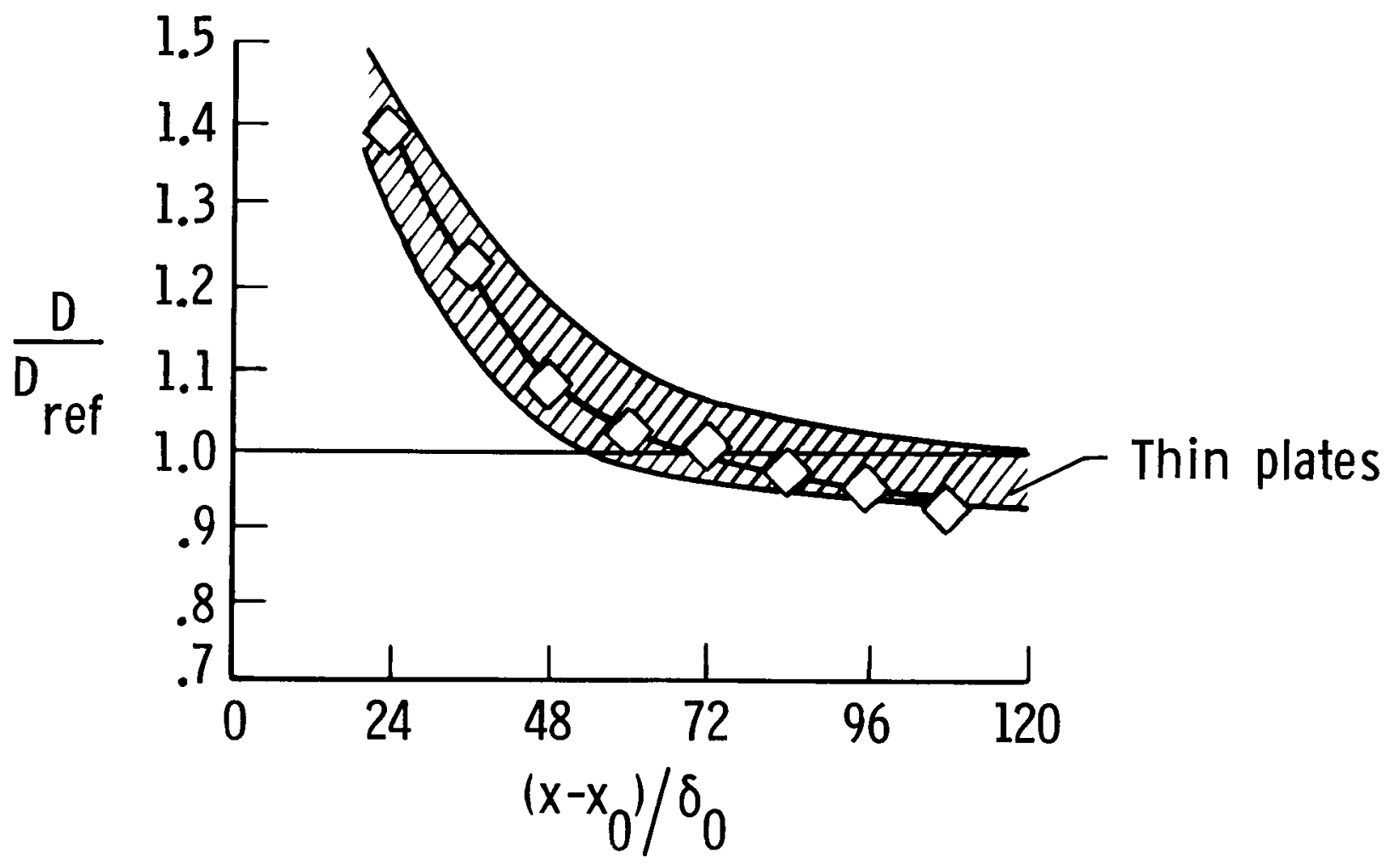




\section{Possible Alternative Uses of LEBU Devices Other Than}

\section{Turbulent Drag Reduction on Smooth Surfaces}

The potential application of these devices to other uses are listed and additional applications will doubtlessly be suggested as research continues. The passive nature of the devices encourages retrofit uses in problems of controlling turbulence.

- Reduce drag on rough surfaces

- Lower radiated turbulent boundary layer noise (possibly less weight of sound insulation in fuselage)

- Lower self-noise on sonar domes/towed arrays

- Increased performance of laser and telescope windows on aircraft fuselages

- Reduce noise signature of torpedoes by reduction of turbulent eddy-propeller interaction

- Reduce size of B. L. diverters on fighter aircraft inlets for lower drag

- Free mixing control (sound/mixing augmen./supp.)

- Combine with riblets (drag reductions add)

- Control of shock-B. L. interaction 


\section{Conclusions}

It has been determined from the present LaRC experiments that tandem, airfoil-shaped LEBU devices can reduce local skin friction as much as 30 percent with a recovery region extending more than 100 boundary layer thicknesses downstream. These airfoils experience near laminar skin friction device drag and produce net drag reductions of up to 7 percent. In contrast to the thin plates used in previous experiments, these airfoils are more than 1000 times stiffer and hence have the potential to withstand the "real" flight environment (dynamic pressure 36 times larger than in low-speed wind tunnels). In addition, the higher Reynolds numbers of the present tests indicate drag reduction performance is at least as good (or better) as at lower Reynolds numbers.

- Symmetric, airfoil-shaped LEBU devices work as well as flat elements - Local $\mathrm{C}_{\mathrm{f}}$ reductions up to $30 \%$

- Reduced $C_{f}$ region extends more than $100 \delta_{0}$

- Net drag reductions up to $7 \%$

- Transonic capable devices (10 times thicker, 1000 times stiffer) - Near laminar skin friction device drag

- LEBU devices work as well (or even better) at high Reynolds numbers as at low Reynolds numbers 